\title{
Accurate, Rapid, High Frequency Empirically Based Predictive Modeling of Arbitrary Geometry Planar Resistive Passive Devices
}

\author{
Ravi Poddar, Member, IEEE, Emily M. Moon, Student Member, IEEE, Martin A. Brooke, Member, IEEE, \\ and Nan Marie Jokerst, Member, IEEE
}

\begin{abstract}
A novel technique is presented for the high speed, accurate, predictive modeling of arbitrary geometry integrated resistor structures manufactured in a variety of technologies, including those of both multichip modules (MCM's) and integrated circuits (IC's). The technique is based upon generating test structures in the process of interest, performing measurements, and extracting the behavior of a few key well identified building blocks. These building blocks can then be used for generating circuit models of other any structure created by valid combinations of those building blocks, which can then be simulated in a standard circuit simulator to predict behavior. The procedure has been experimentally verified, and shows good agreement with actual measurements up to 5-10 GHz. In addition, the model validity has been tested in several circuits by comparing the model predicted results against results obtained using the HP MDS simulator which uses measured parameters directly, with very good results. Since lumped element circuits are generated by this method, structure prediction speed is determined by circuit size and simulator small signal analysis time. The method is versatile and is well suited for circuit design applications.
\end{abstract}

Index Terms - Integrated passives, meander, multichip modules, predictive modeling, resistors, scattering parameters, serpentine, thin film.

\section{INTRODUCTION}

W ITH advances in technology, there is a continuous thrust toward higher levels of system integration and miniaturization. For example, it is now possible to package several integrated circuits together in multichip modules to achieve further compactness and higher performance. With the advent of multichip module and related technologies, such as thick and thin film and multilayer low temperature cofired ceramic (LTCC) processes [1], [2], it is now possible to package a large number of passive components into a small area. There are many advantages to high levels of passive component integration, or simply integration in general; some of the more significant ones are component miniaturization, lower cost, lower power, higher reliability, and higher performance.

Manuscript received May 5, 1997; revised September 22, 1997. This work was supported in part by the National Science Foundation Packaging Research Center under Grant EEC 9402723.

R. Poddar is with Integrated Device Technology, Inc., Duluth, GA 30097 USA.

E. M. Moon, M. A. Brooke, and N. M. Jokerst are with the Georgia Institute of Technology, Atlanta, GA 30332 USA.

Publisher Item Identifier S 1070-9894(98)03736-0.
It is common for high frequency systems to include filters with specifications into the gigahertz range. In order to successfully design a passive filter up to such high frequencies, the behavior of the passive components which comprise the filter must be modeled accurately up to those frequencies. For these complex geometry structures, coupling and parasitics must be taken into account to model nonideal behavior. In this paper, we present an accurate, high speed technique for the predictive modeling of the high frequency behavior of serpentine resistors, suitable for embedding in a multilayer multichip module (MCM), LTCC, or similar process.

\section{MODELING SCHEMES}

High frequency analysis of complex geometrical structures is required to investigate the electrical behavior of designed structures up to a maximum frequency of interest. This analysis is especially important to determine the effects of unwanted spurious couplings and resonances which can greatly affect the overall system response. Analysis of this type is usually only achievable by the use of electromagnetic or RF/microwave simulation tools. There are a number of different solution methodologies available, including the finite and boundary element methods, method of moments (MoM), method of lines, transmission line matrix method, and the finite-difference time domain method, but most of these algorithms are computationally intensive, and are specialized in the area of generating field patterns or scattering parameters [3]-[6]. In addition, the process of obtaining lumped models from these simulators is slow, and is a computationally challenging task. Equivalent circuit models are very useful to a designer who would like to incorporate the complex behavior of the structure in a system, or subsystem level circuit simulation. Currently, most circuit level modeling of passive devices is done after measurements of a particular structure of interest are taken, with the goal of simply having a circuit model of that particular device, with no method of incrementally changing it for different geometries [7]. This could also be accomplished by use of a classical full wave method, but simulation and lumped model extraction would be very time consuming.

A novel method of complex serpentine resistor structure predictive simulation is presented in this paper, based upon the generation of circuit domain passive element models. This procedure involves determining a set of fundamental 
building blocks for the resistor structures, and then designing test structures comprised of combinations of those blocks. The test structures are fabricated and measured up to a desired frequency, and the electrical contribution to the overall response by the building blocks is determined. Equivalent circuits of each of the building blocks are then extracted using a hierarchical extraction procedure that will be described. These building block equivalent circuits can then be used to construct a circuit of any geometry resistor structure that is geometrically comprised of the blocks in a specified way. Simulation of the constructed circuit in a standard SPICE compatible circuit simulator provides an accurate prediction of the behavior of the new structure in a fraction of the time and using far fewer resources than a traditional EM/RF solution methodology. The model of the new structure is verified experimentally by comparing the predicted response with that measured directly from the manufactured structure. It is important to note that the resistor structure that is predicted in this paper is constructed entirely from the building blocks, and the electrical behavior of the structures is based only upon the behavior of the individual building blocks. Using this paradigm, the electrical behavior of any structure which can be constructed appropriately from the defined building blocks can be predicted.

\section{MODELING PROCEDURE}

The first step involved in the resistor modeling procedure was a determination of what types of resistor geometries were to be modeled. Since the theoretical number of possible layouts for a resistor (or any passive structure) is infinite, a restricted set had to be defined in order to determine a sufficiently small set of building blocks that would require characterization. Although at first glance, this type of restriction would seem harsh, it is not impractical. Even with only one line width and interline spacing allowed, a huge array of devices can be designed with large line lengths and many segments. The number of allowed geometries, with different line widths and spacings, can easily be expanded, simply by defining new building blocks. The described procedure is equally applicable to both electrically long and short structures. The reason for this is that as long as the building block models are accurate, they can be connected to model an arbitrarily long piece of material. A well known similar example is the RLCG approximation of a short segment of a transmission line. If each segment is accurate over a fraction of a wavelength, then a long cascade of them can model a structure that is several wavelengths long.

Looking at most designs with integrated passive components, most resistors are laid out in one of two ways; straight lines or serpentine structures, with the former the layout of choice for high frequency applications. For this paper, attention was focused on the serpentine case for several reasons. First, serpentine resistors are more efficient in terms of substrate area when compared to straight lines for the same resistance value, and if modeled correctly and efficiently, may have larger application in the high frequency arena. Secondly, the serpentine structure also presents a more difficult modeling

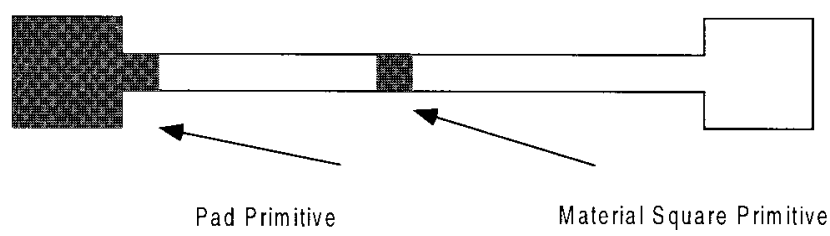

(a)

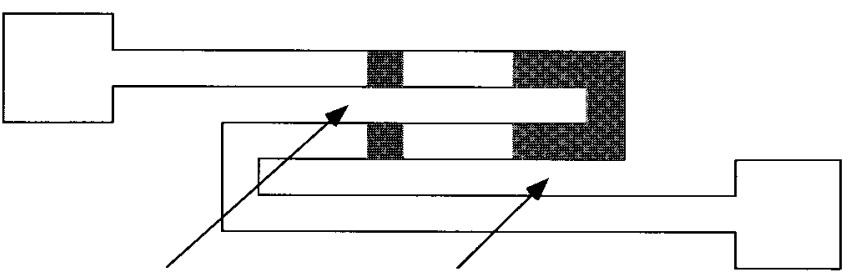

Coupled Square Primitive

U-Shaped Bend Primitive

(b)

Fig. 1. Test structures and associated primitives for serpentine resistor modeling: (a) test structure 1 for modeling of pad and material square primitives and (b) test structure 2 for modeling coupled square and $\mathrm{u}$-shaped bend primitive.

problem due to higher levels of parasitics, such as coupling effects between the segments of the structure, which could considerably affect the overall system response [8].

Resistor modeling with equal line widths and spacing was considered. In the case presented here, $30 \mu \mathrm{m}$ and $40 \mu \mathrm{m}$ line width and spacing resistors were modeled. The serpentine geometry dictated that there were three main fundamental building blocks that required characterization:

1) square building block with connections on opposite sides;

2) u-shaped corner section connecting two parallel segments of the resistor together;

3) coupled block segment to characterize line to line coupling behavior on a per square basis.

Due to the fact that testing of these structures was required, one more building block was added - the probe pad. Coupling is only considered with respect to nearest neighbors, but higher order coupling could be taken into account, however, this would require more test structures and a more complex extraction procedure.

Utilizing custom DC current flow visualization software, the geometry and size of the various building blocks were determined. As mentioned above, four building blocks were defined; an uncoupled square of material, a coupled square, a $u$-shaped bend, and a probe pad. The single square and the coupled square building blocks were only one square in width as expected. The $\mathrm{u}$-shaped block was a total of seven squares in length with the two horizontal sections of the " $u$ " extending for a length of three squares each in order for the current flow distribution to be uniform across the boundaries of the building block. The probe pad primitive was defined to be the size of the pad plus one square of material.

In order to model the four stated building blocks, two test structures were built (Fig. 1). For clarity, the ground lines and pads are not shown in the figure, but the two test structures were designed for compatibility with a ground-signal-ground 
coplanar probe system. The first test structure is simply a line with probe pads on its ends; the purpose of this structure is to help characterize basic uncoupled material parameters, including self resistance, inductance, and capacitance. The second test structure is a 3-segment meander resistor; this structure allows passive characterization of the u-shaped corner segments as well as line to line mutual inductance and coupling capacitance. The structures were characterized using D.C. measurements to determine resistances and network analysis techniques up to $20 \mathrm{GHz}$ to observe parasitic effects in the $S$-parameter response.

\section{PRocessing AND MEASUREMENT}

The test structure resistor material was $\mathrm{Ti} / \mathrm{Au}$ deposited on a $96 \%$ alumina substrate. An electron beam evaporation system was used to deposit $0.04 \mu \mathrm{m}$ of titanium followed by a $0.2 \mu \mathrm{m}$ layer of gold. The thin layer of titanium was used to improve adhesion of the gold to the substrate. Following deposition, the resistors were defined using standard photolithography and etch back. The photoresist was hard baked for 5 min at $125^{\circ} \mathrm{C}$ in order to stabilize it before etching. The gold was etched in a heated $\mathrm{KCN}$ solution for $1 \mathrm{~min}$ followed by a buffered oxide etch to remove the titanium. Due to the surface roughness of the substrate-approximately \pm 1.5 $\mu \mathrm{m}$, the edges of the resistor were jagged, but the lines were continuous. A photograph of the fabricated structures is shown in Fig. 2.

The test structures were measured using network analysis techniques, a DC curve tracer, and a high precision multimeter. For the high frequency measurements, a HP 8510C network analyzer was used in conjunction with a Cascade Microtech probe station and ground-signal-ground configuration probes. Calibration was accomplished using a supplied substrate and utilization of the line-reflect-match (LRM) calibration method. Data was gathered for each of the test structures at over 100 frequency points between $500 \mathrm{MHz}$ and $20 \mathrm{GHz}$ and stored with the aid of computer data acquisition software and equipment. DC I-V measurements of the test structures were also made in order to determine component resistances. At DC, parasitic capacitance and inductance have no effect on the response, and thus the measured resistance values can be used directly in calculating the resistances of the building blocks equivalent circuits.

\section{Modeling And PARAmeter EXtraction}

Circuit models for each of the defined building blocks need to be extracted. The fundamental circuit for the building blocks is based on the partial element equivalent circuit (PEEC) [9] which has been used extensively for interconnect analysis [10] and general three dimensional high frequency structure simulation [11]. Coupling behavior is represented by the coupling capacitance between the center nodes of the two PEEC circuits, as well as by mutual inductances between the left upper and left lower branch inductors in the model, and likewise, for the right hand side. These circuits represent models for the building blocks only; the test structure and resistor circuits are comprised of many of the building

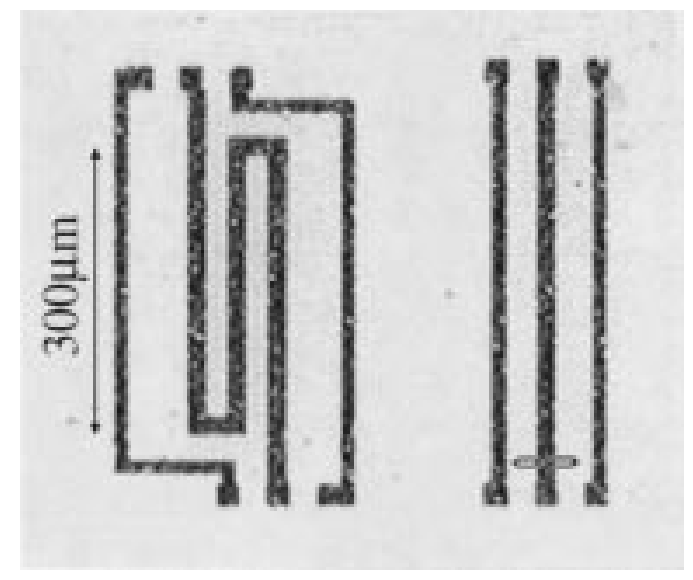

Fig. 2. Fabricated test structure coupon.

block circuits connected in accordance with the structure geometry.

The extraction of the circuit model parameters was achieved in several steps. Due to the highly nonlinear nature of the generated system equations with respect to circuit parameter values, a procedure of hierarchical optimization with respect to measured $S$-parameter and DC resistance data was chosen. The optimization algorithm chosen was Leavenberg-Marquardt [12], and all optimizations and simulations were done using the Hspice circuit simulator on Sun Sparcstation 20 series workstations. The starting point or initial guesses of the circuit parameters were crucial for correct optimization results, and in order to achieve this, an initial optimization was done assuming that each test structure was comprised of just one building block, utilized repetitively across the length of the structure on a per square basis. The initial guess for these circuit parameters were derived from converting the measured $S$-parameters to $Z$-parameters (impedance), and then dividing by the number of blocks used in order to extract the valid $R, L, C$, and $C C$ values for the circuit model. This method was very effective for obtaining a good starting point for the optimizations of the test structure circuits.

The first test structure optimized was structure 1 shown in Fig. 1. The goal was to extract the parameters of the contact pad and the uncoupled material square. The initial guesses were inserted, and the circuit was optimized with respect to measurements up to $10 \mathrm{GHz}$. Once the optimization completed, the computed models were taken and used as valid model parameters for their respective building blocks for test structure 2, shown in Fig. 1. The remaining parameters to be computed for this structure were the line to line coupling parameters (mutual inductance and coupling capacitance), and the parameters for the u-shaped corner. Additionally, line to ground capacitance had to be recomputed for the material square primitive in the presence of adjacent lines. Optimizations were done on measurements performed up to $10 \mathrm{GHz}$. Both optimizations completed with very low residual sum of squares error, indicating accurate results. The modeling results for test structure 1 and 2 are shown in Figs. 3 and 4, respectively. The various circuit models and parameters for the different building blocks are shown in Fig. 5. 


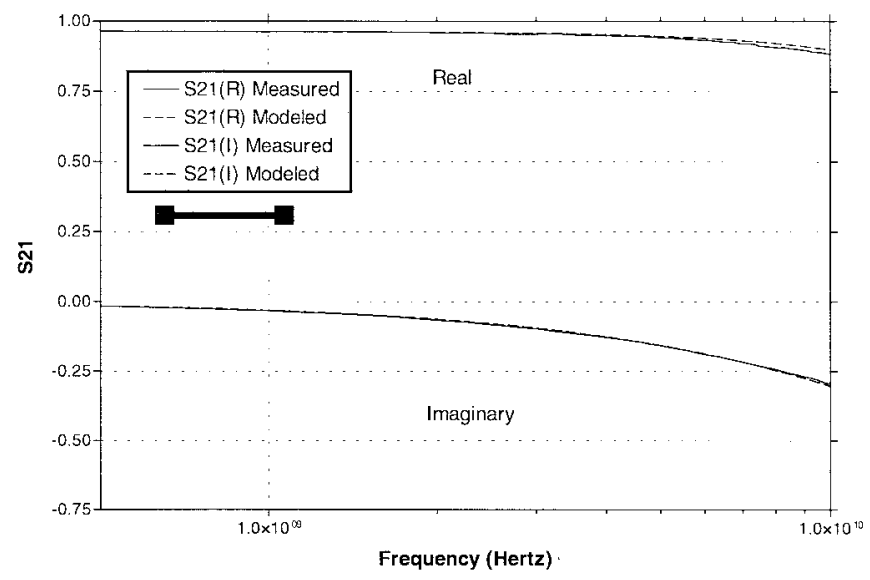

(a)

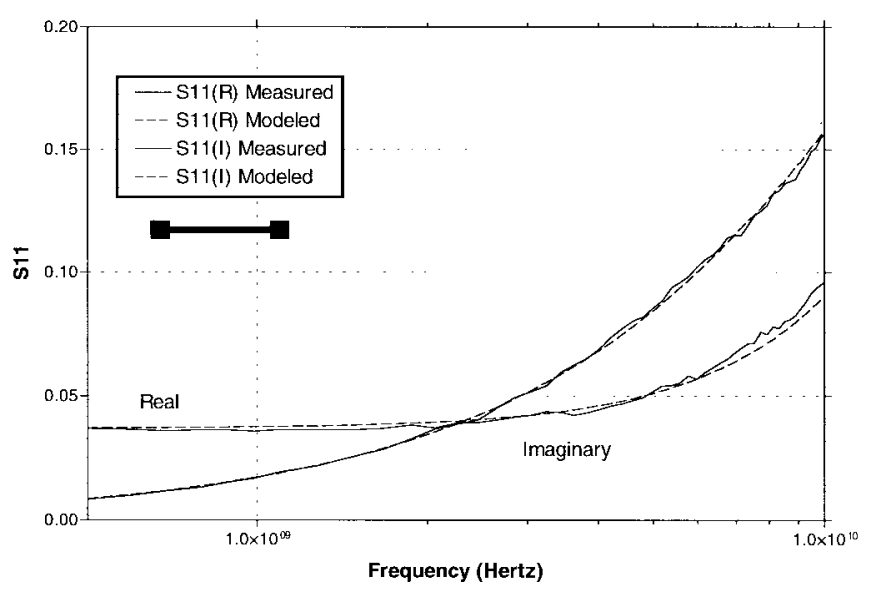

(b)

Fig. 3. Measured versus modeled results for test structure 1: (a) $S_{21}$ real and imaginary response and (b) $S_{11}$ real and imaginary response.

\section{RESULTS}

The extracted fundamental building block models were used to predict the behavior of a nine segment meander resistor which was geometrically comprised of a combination of those blocks. The modeled resistor was then used in several simple circuits to assess the accuracy of the model in common applications. An equivalent circuit of the resistor was constructed by replacing each building block in the structure with its equivalent extracted circuit. Since only first level coupling was taken into account, each material square in each segment of the resistor was coupled to its nearest neighbor by a pair of mutual inductances and a coupling capacitance. As inferred from the circuit description, the resulting circuit of the $30 \mu \mathrm{m}$ line width and $450 \mu \mathrm{m}$ length per segment nine segment resistor was a complex, highly interconnected system, consisting of approximately 700 nodes. The longest path length of the resistor was over 0.65 wavelengths long at $10 \mathrm{GHz}$.

In spite of the large circuit size, AC small signal analysis proceeded very quickly, with the entire circuit simulation completed in under $2 \mathrm{~min}$. The predicted $S$-parameters were compared to measured values of the same structure; the results are shown in Fig. 6. Both real and imaginary parts of $S_{11}$ and $S_{21}$ were well predicted up to $5 \mathrm{GHz}$. In comparison,

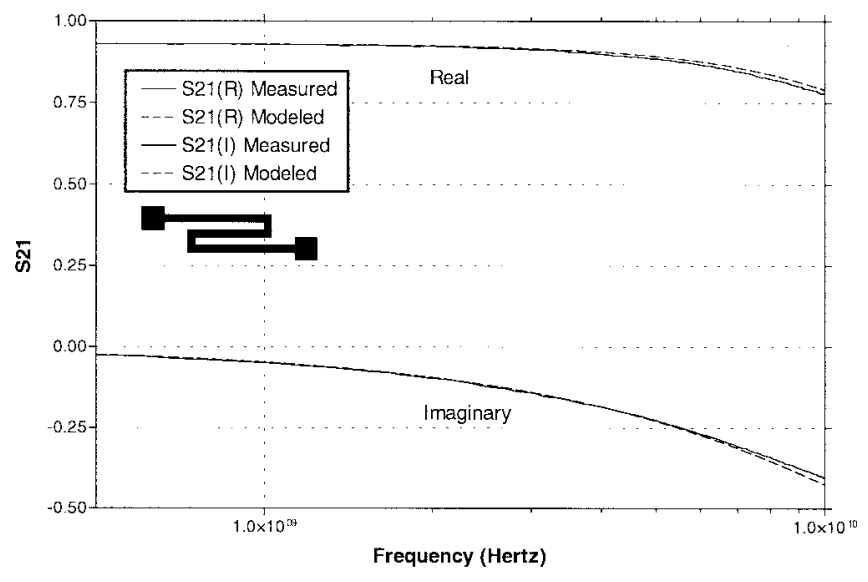

(a)

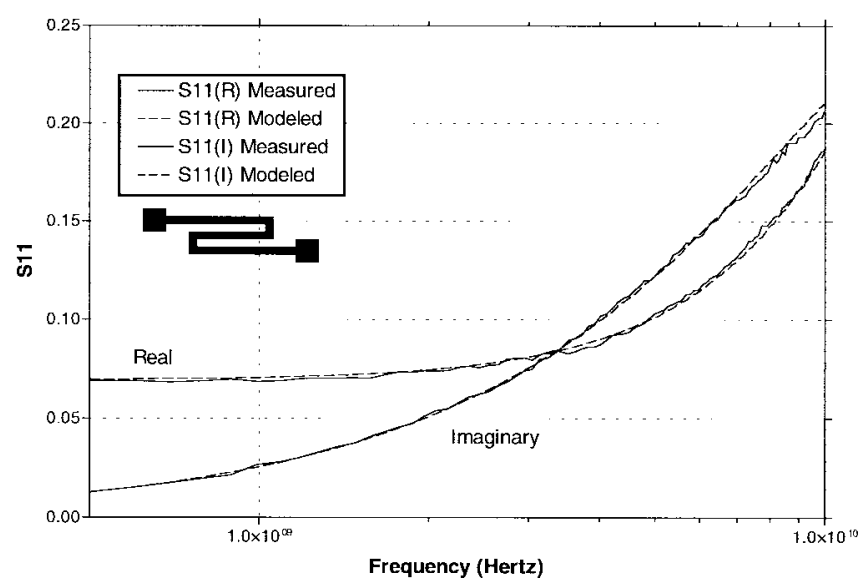

(b)

Fig. 4. Measured versus modeled results for test structure 2: (a) $S_{21}$ real and imaginary response and (b) $S_{11}$ real and imaginary response.

the same structure was designed and simulated in a method of moments solver with a $3 \mathrm{GHz}$ meshing frequency. The structure required 72 min to complete, while consuming approximately $50 \mathrm{MB}$ of system memory and utilizing two processors in a multiprocessing Sun workstation. Thus, for this example, a speedup factor of approximately 35 was obtained. For more complex structures, simulation time of the method of moments solver would increase dramatically, whereas using our approach, simulation time would increase only with the number of elements in the equivalent circuit.

The resistor model was tested in a six segment LC circuit, with the resistor used as a termination. The inductance and capacitance were chosen such that a characteristic impedance per segment of $50 \Omega$ was obtained. The circuit is shown in Fig. 7(a). The circuit was simulated in both MDS (using data measured from the fabricated resistor) and in the circuit simulator (using the model constructed only from building blocks), two port $S$-parameter simulation were predicted. The results of the simulation are shown in Fig. 7(b) and (c). Both $S_{11}$ and $S_{21}$, real and imaginary parts are predicted well up to $20 \mathrm{GHz}$. The results show that the resistor behavior has been modeled well using the described method in actual circuit simulation applications. 

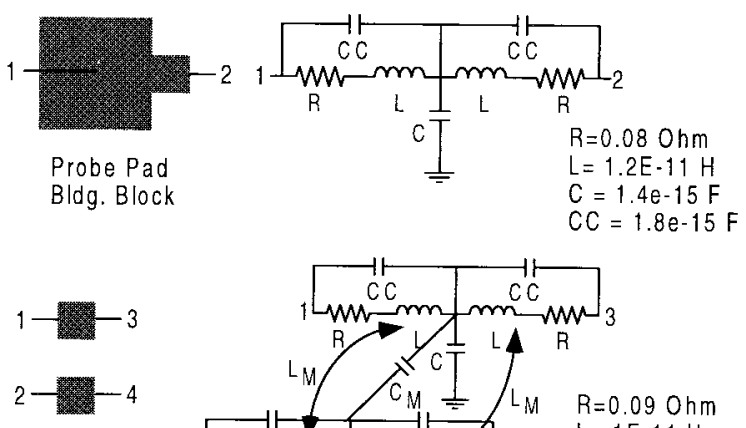

Coupled Line Bldg. Block
$C=1.4 \mathrm{e}-15 \mathrm{~F}$

(1)
$\mathrm{R}=0.09 \mathrm{Ohm}$ $L=1 \mathrm{E}-11 \mathrm{H}$

$\mathrm{C}=1.1 \mathrm{e}-15 \mathrm{~F}$

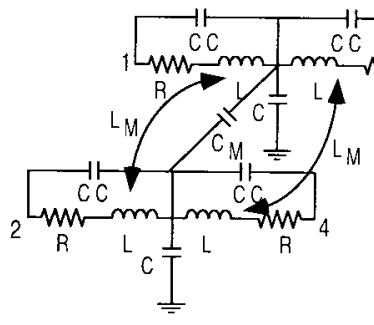

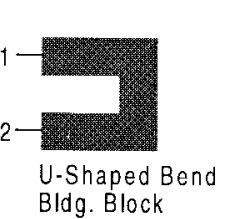
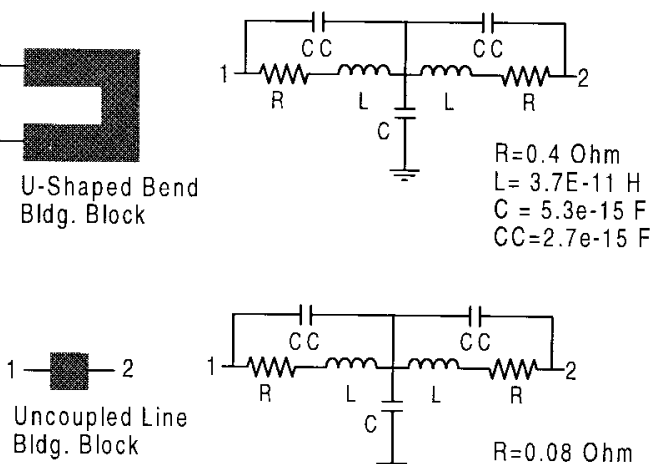

$C M=0.4 e-15 F$

$C C=1.4 \mathrm{e}-15 \mathrm{~F}$

$\mathrm{LM}=0.20$

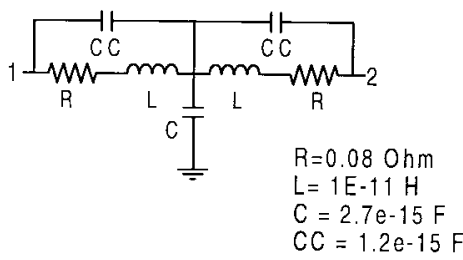

Fig. 5. Building blocks with associated circuit topologies and model parameters.

\section{DISCUSSION}

It is important to note that all the presented results and the model for the nine segment resistor are based upon the four building block models extracted from only two measured test structures. Although the number of building blocks in this case is small, a huge variety of different resistor designs can still be predicted with this set, the only restrictions are that the same line width and spacing be used, in this case $30 \mu \mathrm{m}$, but the length and number of segments of the resistor are unconstrained. Various other line width and spacing combinations can easily be added, simply be defining additional building blocks and test structures.

The circuit model for the nine segment resistor was created entirely from the building block equivalent circuits. The behavior prediction for a terminated LC circuit was also obtained by using these building blocks to create equivalent circuit representations of the various systems, followed by simulation in a circuit simulator to achieve results. The resistor modeling and simulation process is quick, and is particularly useful and efficient in the area of circuit design, where a designer can easily modify a resistor structure by adding or removing building blocks, and can quickly observe the resulting effect on the overall system response. Essentially, the proposed method gives the designer freedom to optimize or "tune" the passive resistive components in order to meet design goals. In particular, the overall shape of the resistor can be essentially arbitrary to make effective use of module space.

The main limitation of this method is the ability to obtain good models of the building blocks. In the work presented here, nonlinear effects such as skin effect are not included in the building block models, and hence such phenomena are not modeled. Additionally, only first order coupling distances are considered, so larger coupling distance effects are neglected, which could produce error at higher frequencies. In general, if a circuit or any other type of model, such as a behavioral model or a direct $S$-parameter data model of the building blocks could be obtained with complete accuracy over the frequency range of interest, then accurate simulations of any design rule compliant structure comprised of those building blocks are obtainable with this method. A second limitation of the method

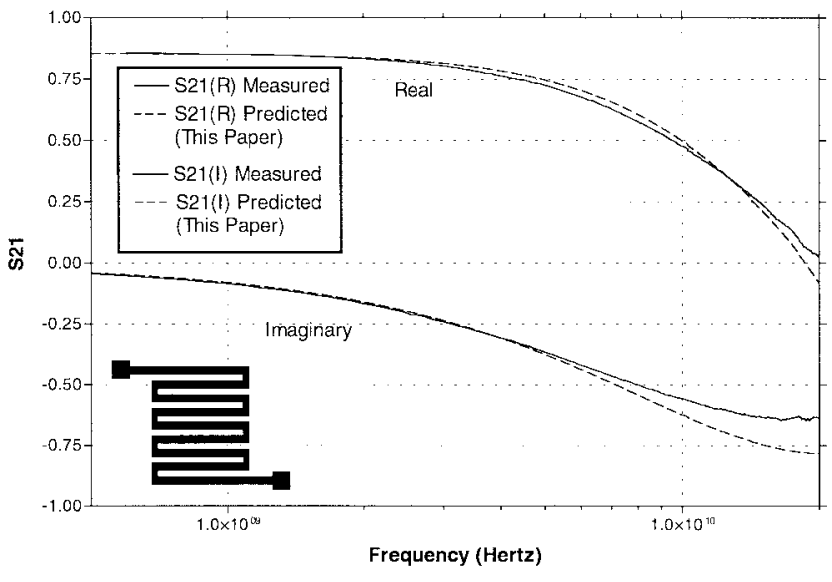

(a)

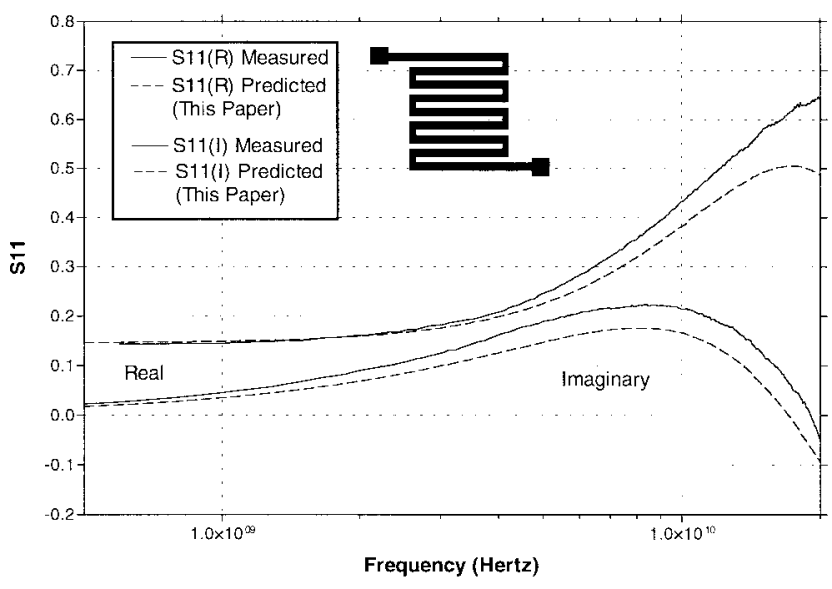

(b)

Fig. 6. Measured versus predicted results for 9 segment resistor: (a) $S_{21}$ real and imaginary response and (b) $S_{11}$ real and imaginary response.

lies in the area of test structure design. Test structures must be designed such that the contributions of all of the building blocks can be de-embedded. The issue in this case is that the measurement equipment must be sensitive enough to detect incremental changes in the output response due to particular building blocks. In the work presented here, we have found 


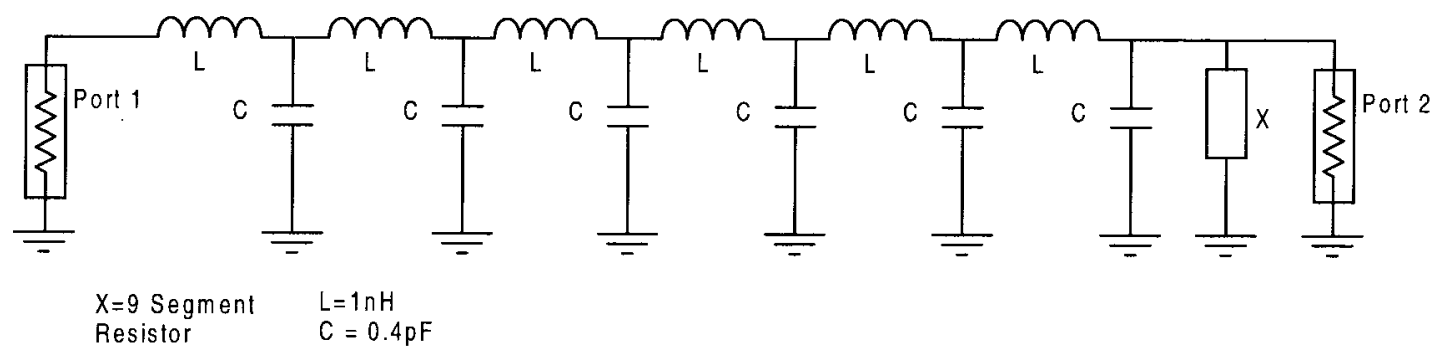

(a)

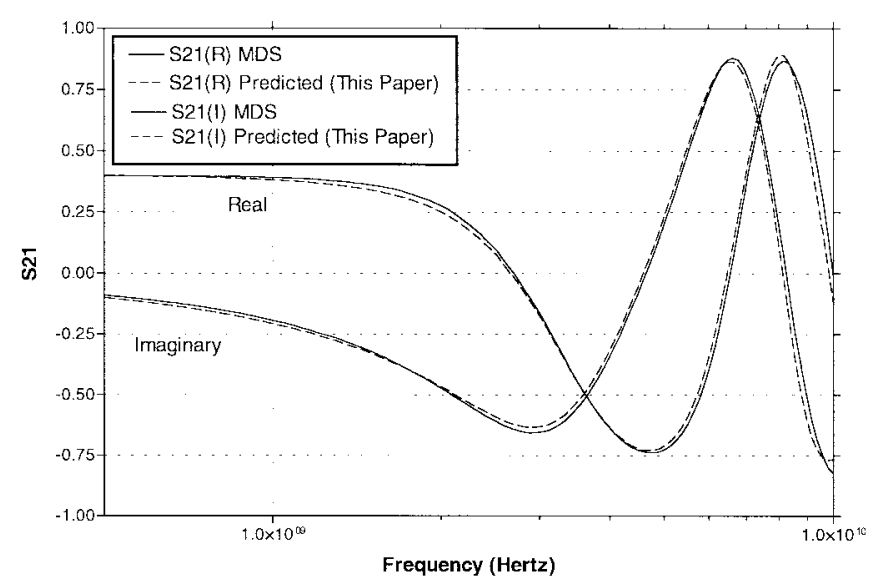

(b)

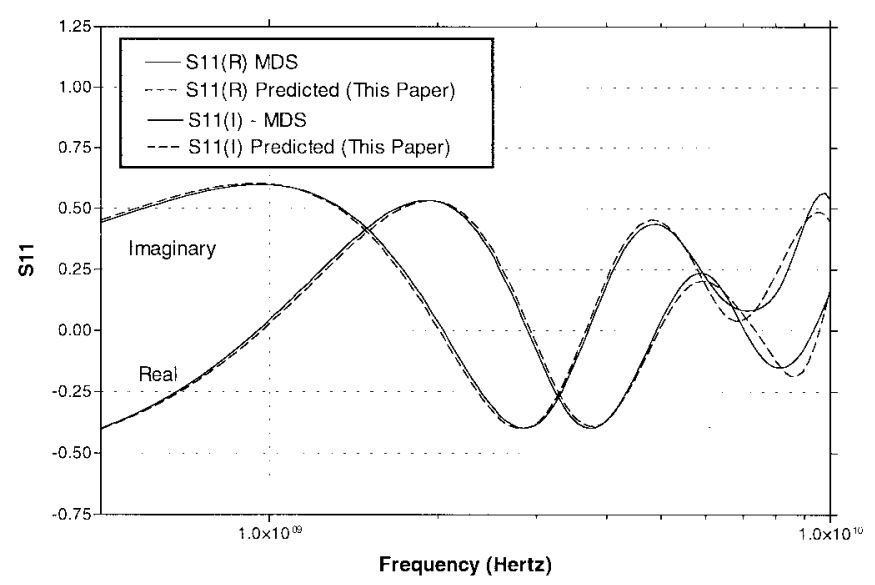

(c)

Fig. 7. MDS generated versus predicted results for 6-segment LC circuit with resistive termination: (a) circuit diagram, (b) $S_{21}$ real and imaginary response, and (c) $S_{11}$ real and imaginary response.

that well calibrated network analysis equipment is extremely sensitive, and that this issue did not pose any problems. Neither of these limitations were encountered in the work presented in this paper.

\section{CONCLUSION}

A fast, accurate, method for the high frequency behavioral prediction of serpentine resistors has been described. The procedure requires design and fabrication of test structures in order to characterize models for certain well defined geometric building blocks. These building blocks have design rules associated with them, and these in turn dictate design restrictions for resistor designs in order to be correctly modeled. Resistors designed with the correct building blocks have been modeled accurately into the gigahertz frequency range, with simulation speeds far greater than that of conventional electromagnetic or RF simulators. This procedure creates highly flexible wide band circuit level models of the resistors, which are extremely useful since they can be incorporated into the circuit design stage to investigate how they will affect overall system performance. This method shows potential for modeling other kinds of passive devices as well, such as capacitors and inductors, and applications in the area of integrated circuit interconnect analysis are also possible.

\section{ACKNOWLEDGMENT}

The authors would like to thank the Cleanroom Staff, Georgia Tech Microelectronics Research Center, for their assistance in this work.

\section{REFERENCES}

[1] R. Tummala and E. J. Rymaszewski, Microelectronics Packaging Handbook. New York: Van Nostrand Reinhold, 1989.

[2] R. L. Brown and A. A. Shapiro, "Integrated passive components and MCM's: The future of microelectronics," in Proc. Int. Conf. Exhibition Multichip Modules, Apr. 1993, pp. 287-94.

[3] A. Nakatani, S. A. Maas, and J. Castaneda, "Modeling of high frequency MMIC passive components," IEEE MTT-S Int. Microwave Symp. Dig., pp. 1139-1141, June 1989.

[4] K. Naishadham and T. W. Nuteson, "Efficient analysis of passive microstrip elements in MMIC's," Int. J. Microwave Millimeter-Wave Computer-Aided Eng., vol. 4. no. 3, pp. 148-62, Apr. 1994.

[5] R. Sorrentino, "Numerical methods for passive components," IEEE MTT-S Int. Microwave Symp. Dig., pp. 619-622, 1988.

[6] D. G. Swanson, Jr., "Simulating EM fields," IEEE Spectrum, vol. 28 , pp. 34-37, Nov. 1991.

[7] V. K. Sadhir, I. J. Bahl, and D. A. Willems, "CAD compatible accurate models of microwave passive lumped elements for MMIC applications," Int. J. Microwave Millimeter-Wave Computer-Aided Eng., vol. 4, no. 2, pp. 148-62, Apr. 1994.

[8] W. H. Haydl, "Properties of meander coplanar transmission lines," IEEE Microwave Guided Wave Lett., vol. 2 pp. 439-441, Nov. 1992.

[9] A. E. Ruehli, "Equivalent circuit models for three dimensional multiconductor systems," IEEE Trans. Microwave Theory Tech., vol. MTT-22, pp. 216-221, Mar. 1974. 
[10] H. Heeb and A. E. Ruehli, "Three-dimensional interconnect analysis using partial element equivalent circuits," IEEE Trans. Circuits Syst. I, vol. 39, pp. 974-982, Nov. 1992.

[11] A. E. Ruehli and H. Heeb, "Circuit models for three-dimensional geometries including dielectrics," IEEE Trans. Microwave Theory Tech., vol. 40, pp. 1507-1516, July 1992.

[12] W. H. Press, B. P. Flannery, S. A. Teukolsky, and W. T. Vetterling, Numerical Recipes in C, The Art of Scientific Computing. New York: Cambridge University Press, 1988.

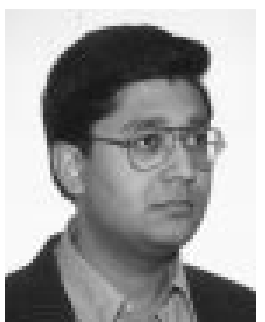

Ravi Poddar (S'89-M'96) received the B.S.E.E (with highest honors) and M.S.E.E. degrees in 1991 and 1995, respectively, and the Ph.D. degree in electrical and computer engineering from the Georgia Institute of Technology, Atlanta, in 1998.

$\mathrm{He}$ is with Integrated Device Technology, Inc., Duluth, GA, working in the area of integrated circuit physical extraction and verification. His research interests include high frequency, computer aided design and modeling of integrated passive devices and multichip modules, high frequency modeling of deep submicron integrated circuit interconnects, CMOS transistor modeling, statistical analysis and yield prediction, and high speed automated test and measurement techniques.

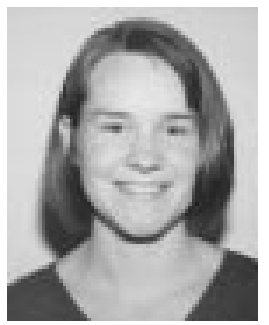

Emily M. Moon (S'97) received the A.B. degree in physics from Smith College, Northampton, MA, in 1996 and is currently pursuing the M.S. degree in electrical engineering from the Georgia Institute of Technology, Atlanta.

Her area of research is in high-speed optoelectronics and three-dimensional optical interconnects.
Martin A. Brooke ( $\left.\mathrm{S}^{\prime} 85-\mathrm{M}^{\prime} 87\right)$ received the B.E. degree in electronics from Auckland University, Auckland, New Zealand, in 1981, and the M.S and Ph.D. degrees in electrical engineering from the University of Southern California, Los Angeles, in 1984 and 1988, respectively.

$\mathrm{He}$ is the Analog Device Career Development Assistant Professor of Electrical Engineering at the Georgia Institute of Technology, Atlanta, and is developing electronically adjustable parallel analog circuit building blocks that achieve high levels of performance and fault tolerance. His current research interests are in high-speed high-precision signal processing. Current projects include development of adaptive neural network and analog multipliers and dividers, precision analog amplifiers, communications circuits, and sensor signal processing circuitry. To support this large analog and digital systems research, he actively pursues systems level circuit modeling research. He has developed software that reduces the complexity of large analog electronic system models to a user specified tolerance.

Dr. Brooke received the NSF Research Initiation Award in 1990.

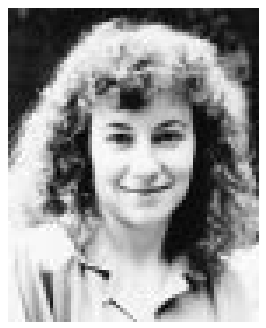

Nan Marie Jokerst (S'83-M'88) received the B.S degree in physics and mathematics from Creighton University, Omaha, NE, in 1982, and the M.S. and $\mathrm{Ph} . \mathrm{D}$. degrees in electrical engineering from the University of Southern California, Los Angeles, in 1984 and 1989, respectively.

Her research work has focused on fabrication, integration, and application of thin film semiconductor materials and devices. She has over 100 refereed publications and presentations, and three patents.

Dr. Jokerst received the Newport Research Award in 1986, the Award for Best Teacher in Electrical Engineering at Georgia Tech in 1990, and was a Hewlett Packard Fellow in 1983. She was named a National Science Foundation Presidential Young Investigator in 1990 and a DuPont Young Professor in 1989. She is a member of the Optical Society of America. She has served as Secretary, Treasurer, Vice Chair, and Chair of the Atlanta IEEE Section. She is the Chair of the OSA Engineering Council. She is on the IEEE LEOS Board of Governors as an Elected Member and as the Vice President for Conferences. She has served on numerous program committees for OSA and IEEE LEOS. 